\title{
Outage Probability of Beamforming for Multiuser MIMO Relay Networks with Interference
}

\author{
$\begin{array}{lll}\text { S. Zhou } & \text { G. Alfano } & \text { C.-F.Chiasserini } \\ * & \text { A. Nordio }\end{array}$
}

\begin{abstract}
We investigate the outage probability of a dual-hop MIMO system where amplifyand-forward relay and beamforming are used. A practically important case where proportional fair scheduling is implemented to schedule users transmissions and co-channel interference exists is considered in the analysis. Specifically, taking into account Rayleigh fading and the path-loss effect, we derive the lower bound and the asymptotic expressions of the outage probability in closed form, with arbitrary number of antennas and users. The proposed results are validated via Monte Carlo simulations.
\end{abstract}

\section{INTRODUCTION}

Cooperative multiple-input multiple-output (MIMO) relay networks have been extensively investigated for the last decade from both industry and academia since they are able to improve the throughput and broaden the coverage of wireless communication systems. In particular, multiuser relay networks (MRN) have been recently proposed and adopted in some relevant standards such as IEEE $802.16 \mathrm{~m}$ and LTE-A.

In MRN a source $(s)$ is assisted by a relay $(r)$ to send information to a set of users $\mathcal{U}=\{1, \ldots, U\}$. The user to be served is selected according to a scheduling strategy that aims at reaping the benefits of multiuser diversity. In particular, we assume that the well-known proportional fair scheduling (PFS) strategy is adopted [1]. Such strategy assigns each user a scheduling priority that is proportional to the user relative condition in terms of signal-to-noise-ratio (SNR).

We remark that most existing works assume that at least one node of the network is equipped with single antenna and the path-loss effect can be neglected. In this work, we address a cellular MIMO network where a base station transmits data to multiple users via a relay node. The relay node schedules the second-hop transmission by implementing PFS strategy. In such dual-hop MIMO MRN, we provide a comprehensive outage analysis of the coupled effects of antenna diversity, path-loss, multiuser diversity, and co-channel interference (CCI). The remainder of the paper is organized as follows.

\footnotetext{
*DET, Politecnico di Torino, C.so Duca degli Abruzzi 24, 10129 Torino, Italy, e-mail: \{siyuan.zhou, alfano, chiasserini\}@polito.it.

${ }^{\dagger}$ CNR-IEIIT, C.so Duca degli Abruzzi 24, 10129 Torino, Italy, e-mail: alessandro.nordio@polito.it
}

In Section II, the network model is introduced. Section III first investigates the distribution of the signal-to-interference-plus-noise-ratio (SINR) over each hop. Then the lower bound and high-SNR asymptotic expressions on the system outage probability are presented. Section IV provides numerical results while Section V concludes the paper.

\section{SYSTEM MODEL}

We assume that communications are subject to CCI and all nodes (source $s$, relay $r$, interferers, and users $u \in \mathcal{U}$ ) operate in half-duplex mode. Also, no direct link exists between the source and the users. Source and relays are equipped with $n_{s}$ and $n_{r}$ antennas, respectively, while users are equipped with $n_{d}$ antennas. Thanks to the help of the relay, the source transfers information to the users, whose number is denoted by $U$. In the first hop, the received signal at the relay suffers the CCI coming from a set of out-of-cell interferers denoted by $\mathcal{S}_{r}$. In the second hop, only one out of the $U$ users is scheduled for reception in each scheduling period. Since users are located at different points within the cell range, they may be affected by different sets of out-of-cell interferers. If user $u \in \mathcal{U}$ is selected by the scheduler, we denote by $\mathcal{S}_{u}$ the set of co-channel interferers affecting the received signal.

Perfect channel state information (CSI) related to the two hops is available at source, relay and users. Thus, MIMO beamforming can be used for communication.

Signal propagation is assumed to be affected by both large-scale path-loss and small-scale uncorrelated Rayleigh fading. It is fair to assume that the signal received by antennas belonging to the same array experiences the same path loss. Specifically, the channel gain from transmitter $i(i \in\{s, r\})$ to receiver $j(j \in\{r\} \cup \mathcal{U})$ is given by $\sqrt{d_{i j}^{-a_{i j}}} \mathbf{H}_{i j}$, where $d_{i j}, a_{i j}$, and $\mathbf{H}_{i j}$ represent the mutual node distance, the path-loss exponent, and the channel matrix, respectively. Under the Rayleigh fading assumption, each element of $\mathbf{H}_{i j}$ is Gaussian distributed with zero mean and unit variance. In the following $\mathbf{H}_{i j}=\mathbf{U}_{i j} \boldsymbol{\Lambda}_{i j} \mathbf{V}_{i j}^{\dagger}$ indicates the singular value decomposition of $\mathbf{H}_{i j}$, where $\boldsymbol{\Lambda}_{i j}$ is the diagonal matrix containing the channel singular values.

Also, we denote the product of the transmit 
power and the path-loss attenuation from $i$ to $j$ by $\alpha_{i j}=P_{i} d_{i j}^{-a_{i j}}$.

In the first hop, the source $s$ transmits the random information symbol $x$, which is assumed to have zero mean and variance $\mathbb{E}\left[|x|^{2}\right]=1$, while the $k$-th interferer in $\mathcal{S}_{r}$ transmits the zero-mean unit-variance random symbol $x_{k}$. We consider that the maximum ratio transmission (MRT) technique [2] is used at the transmitters. Thus, for each transmitter-receiver pair, $(i, j), i$ precodes the symbol using the right singular vector $\mathbf{v}_{i j}$ of the channel matrix $\mathbf{H}_{i j}$, which corresponds to the maximum singular value $\sqrt{\lambda_{j}}$. Then the receiver filters the received signal by using the left singular vector $\mathbf{u}_{i j}$ of $\mathbf{H}_{i j}$, which corresponds to the maximum singular value. Therefore, the received signal at the relay $r$ is a combination of the desired beamforming signal, the interfering signals, and the AWGN $z_{r}$, i.e.,

$$
\begin{aligned}
y_{r} & =\alpha_{s r}^{\frac{1}{2}} \mathbf{u}_{s r}^{\mathrm{H}} \mathbf{H}_{s r} \mathbf{v}_{s r} x+\sum_{k \in \mathcal{S}_{r}} \alpha_{k r}^{\frac{1}{2}} \mathbf{u}_{s r}^{\mathrm{H}} \mathbf{H}_{k r} \mathbf{v}_{k r} x_{k}+z_{r} \\
& =\left(\alpha_{s r} \lambda_{r}\right)^{\frac{1}{2}} x+\sum_{k \in \mathcal{S}_{r}} \alpha_{k r}^{\frac{1}{2}} h_{k r} x_{k}+z_{r}
\end{aligned}
$$

where $\sqrt{\lambda_{r}}=\mathbf{u}_{s r}^{\mathrm{H}} \mathbf{H}_{s r} \mathbf{v}_{s r}$ and $h_{k r}=\mathbf{u}_{s r}^{\mathrm{H}} \mathbf{H}_{k r} \mathbf{v}_{k r}$. It is easy to see that the coefficients $h_{k r}$, with $k \in$ $\mathcal{S}_{r}$, are independent zero-mean complex Gaussian variables [4].

In the second hop, let us assume that the $u$-th user is scheduled for transmission. The relay node first amplifies the received signal $y_{r}$ with an adaptive gain $g$, which is set so as to normalize the signal power. Specifically,

$$
G=\sqrt{\frac{P_{r}}{\alpha_{s r} \lambda_{r}+\sum_{k \in \mathcal{S}_{r}} \alpha_{s r}\left|h_{k r}\right|^{2}+1}} .
$$

Then the relay forwards the signal to the $u$-th user by beamforming it through the channel characterized by matrix $\mathbf{H}_{r u}$. Again, assuming that the relay precodes the signal according to the MRT technique, the received signal at the $u$-th user can be written as:

$$
y_{u}=G d_{r u}^{-\frac{a_{r u}}{2}} \lambda_{u}^{\frac{1}{2}} y_{r}+\sum_{l \in \mathcal{S}_{u}} \alpha_{l u}^{\frac{1}{2}} \mathbf{u}_{r u}^{\mathrm{H}} \mathbf{H}_{l u} \mathbf{v}_{l u} x_{l}+z_{u} .
$$

In (2), similarly to the first hop, $\lambda_{u}$ is the largest eigenvalue of $\mathbf{H}_{r u}$, and $z_{u}$ is a white noise with zero mean and unit variance. From now on, we define and $h_{l u} \triangleq \mathbf{u}_{r u}^{\mathrm{H}} \mathbf{H}_{l u} \mathbf{v}_{l u}$. Similarly to $h_{k r}$ 's, $h_{l u}$ 's, with $l \in \mathcal{S}_{u}$, are independent zero-mean complex Gaussian variables.

Let us define $\sigma_{r}=\alpha_{s r} \lambda_{r}$ and $\sigma_{u}=\alpha_{r u} \lambda_{u}$ as the instantaneous SNRs of the two hops. According to the PFS scheme, the scheduler grants access to the user that experiences the best relative condition in terms of SNR. The selected user is

$$
u^{\star}=\arg \max _{u \in \mathcal{U}} \frac{\sigma_{u}}{\bar{\sigma}_{u}}
$$

where $\bar{\sigma}_{u}$ is the average SNR of the $u$-th user. Note that $\bar{\sigma}_{u}=\mathbb{E}\left[\sigma_{u}\right]=\alpha_{r u} \mathbb{E}\left[\lambda_{u}\right]$, thus the above ratio does not depend on $\alpha_{r u}$. Moreover, since all channel matrices are identically distributed, $\mathbb{E}\left[\lambda_{u}\right]=$ $\mathbb{E}[\lambda]$, for all $u \in \mathcal{U}$. Then

$$
u^{\star}=\arg \max _{u \in \mathcal{U}} \lambda_{u} .
$$

We assume that the user and the corresponding interferers are stationary during the duration of the transmission. Thus, the SNR of the selected user, $\sigma_{\star}$, is given by

$$
\sigma_{\star}=\alpha_{r u^{*}} \lambda_{u^{\star}} .
$$

\section{OUTAGE PROBABILITY}

The outage probability of the system is defined as the probability that the end-to-end SINR falls below a predefined threshold. The instantaneous endto-end SINR of the system can be written as

$$
\gamma=\frac{\gamma_{r} \gamma_{\star}}{\gamma_{r}+\gamma_{\star}+1}
$$

where $\gamma_{r}=\sigma_{r} /(\beta+1)$ is the SINR at the relay, with $\beta=\sum_{k \in \mathcal{S}_{r}} \alpha_{k r}\left|h_{k r}\right|^{2}$. Also $\gamma_{\star}=\sigma_{\star} /\left(\mu_{\star}+\right.$ 1) is the SINR at the served user, with $\mu_{\star}=\mu_{u^{\star}}$ and $\mu_{u}=\sum_{l \in \mathcal{S}_{u}} \alpha_{l u}\left|h_{l u}\right|^{2}$. Note that $\mu^{\star}$ denotes the power of the CCI at the served user. In the following, the statistical distributions of $\gamma_{r}$ and $\gamma_{\star}$ are investigated separately and a lower bound to the system outage probability is derived.

Indeed, the analytical expression for the cumulative density function (CDF) of $\gamma$ is currently unavailable. However, a mathematically tractable upper bound of $\gamma$ can be expressed as

$$
\gamma=\frac{\gamma_{r} \gamma_{\star}}{\gamma_{r}+\gamma_{\star}+1}<\min \left(\gamma_{r}, \gamma_{\star}\right)=\gamma_{U B} .
$$

Then, thanks to the independence between $\gamma_{r}$ and $\gamma_{\star}$, the outage probability of $\gamma$ can be lower bounded by

$$
\begin{aligned}
P_{\text {out }}^{L B}(x) & =P\left(\gamma_{U B}<x\right) \\
& =1-\left(1-F_{r}(x)\right)\left(1-F_{\star}(x)\right) .
\end{aligned}
$$

In order to derive the CDF of $\gamma_{r}$ and $\gamma_{\star}$, we proceed as follows. Let us define $t=\min \left(n_{s}, n_{r}\right)$, $v=\max \left(n_{s}, n_{r}\right), \mathbf{A}=\operatorname{diag}\left(\alpha_{1 r}, \alpha_{2 r}, \ldots, \alpha_{\left|\mathcal{S}_{r}\right| r}\right)$, $\varrho(\mathbf{A})$ is the number of distinct diagonal elements 
of $\mathbf{A}, \alpha_{\langle 1 r\rangle}>\alpha_{\langle 2 r\rangle}>\cdots>\alpha_{\langle\varrho(\mathbf{A}) r\rangle}$ are the distinct diagonal elements in decreasing order, $\tau_{i}(\mathbf{A})$ is the multiplicity of $\alpha_{\langle i r\rangle}$, and $\chi_{i j}(\mathbf{A})$ is the $(i, j)$ th characteristic coefficient of $\mathbf{A}$ [4]. Since $\gamma_{r}=$ $\sigma_{r} /(\beta+1)$, the closed-form CDF expression of $\gamma_{r}$ for the source-relay hop with beamforming is given by

$$
F_{r}(x)=\int_{0}^{\infty} p_{\beta}(y) F_{\sigma_{r}}(x(1+y)) \mathrm{d} y .
$$

The expression of the density $p_{\beta}(y)$ can be found in [4, eq.(29)] while the $\operatorname{CDF} F_{\sigma_{r}}(x(1+\beta))$ is given in $[4$, eq.(30)]. Using the above results, we obtain

$$
F_{r}(x)=1-\sum_{\mathbf{w}} \epsilon_{r}(\mathbf{w}) x^{m} \frac{\mathrm{e}^{-b x / \alpha_{s r}}}{\left(1+\frac{\alpha_{\langle i r\rangle} b}{\alpha_{s r}} x\right)^{g+j}}
$$

where

$$
\sum_{\mathbf{w}} \triangleq \sum_{i=1}^{\varrho(\mathbf{A})} \sum_{j=1}^{\tau_{i}(\mathbf{A})} \sum_{b=1}^{t} \sum_{c=v-t}^{(v+t) b-2 b^{2}} \sum_{m=0}^{c} \sum_{g=0}^{m}
$$

and

$$
\epsilon_{r}(\mathbf{w})=\frac{\left(\begin{array}{c}
m \\
g
\end{array}\right) d(b, c)}{(j-1) ! m !}\left(\frac{b}{\alpha_{s r}}\right)^{m} \chi_{i j}(\mathbf{A}) \Gamma(g+j) \alpha_{\langle i r\rangle}^{g}
$$

where $d(b, c)$ is the coefficient that can be precomputed with an efficient algorithm given in [3]. The proof is given in our technical report [5].

The characterization of the distribution of $\gamma^{\star}$ depends on the adopted multiuser scheduling algorithms. Indeed, the CDF of $\gamma^{\star}$ is written as

$$
F_{\star}(x)=\sum_{u=1}^{U} \mathbb{P}\left(u^{\star}=u\right) \mathbb{P}\left(\gamma_{u} \leq x \mid u=u^{*}\right)
$$

where $\gamma_{u}=\sigma_{u} /\left(\mu_{u}+1\right)$. In the PFS scheme, each user is scheduled with the same probability, i.e., $\mathbb{P}\left(u^{\star}=u\right)=1 / U$. Furthermore,

$$
\begin{aligned}
\mathbb{P}\left(\gamma_{u} \leq x \mid u=u^{*}\right) & =\int_{0}^{\infty} p_{\mu_{u}}(y) F_{\lambda_{u}^{\star}}\left(\frac{x(1+y)}{\alpha_{r u}}\right) \mathrm{d} y \\
& =\int_{0}^{\infty} p_{\mu_{u}}(y) F_{\lambda_{u}}\left(\frac{x(1+y)}{\alpha_{r u}}\right) \mathrm{d} y(9)
\end{aligned}
$$

where we exploited the fact that $\lambda_{u^{\star}}=\max _{u \in \mathcal{U}} \lambda_{u}$ and that all $\lambda_{u}$ are i.i.d. Next, we denote by $q=\min \left(n_{r}, n_{d}\right), p=\max \left(n_{r}, n_{d}\right), \mathbf{B}_{u}=$ $\operatorname{diag}\left(\alpha_{1 u}, \alpha_{2 u}, \ldots, \alpha_{\left|\mathcal{S}_{u}\right| u}\right), \varrho\left(\mathbf{B}_{u}\right)$ is the number of distinct diagonal elements of $\mathbf{B}_{u}, \alpha_{\langle 1 u\rangle}>\alpha_{\langle 2 u\rangle}>$ $\cdots>\alpha_{\left\langle\varrho\left(\mathbf{B}_{u}\right) u\right\rangle}$ are the distinct diagonal elements in decreasing order, $\tau_{i}\left(\mathbf{B}_{u}\right)$ is the multiplicity of $\alpha_{\langle i u\rangle}$, and $\chi_{i j}\left(\mathbf{B}_{u}\right)$ is the $(i, j)$-th characteristic coefficient of $\mathbf{B}_{u}$ [4]. Following the same steps as above, we can derive the closed-form CDF expression of $\gamma^{\star}$, under the PFS scheme as follows. The density $p_{\mu_{u}}(y)$ can be written as in [4, eq.(29)] while the expression for $F_{\lambda_{u}}(y)$ can be found in [4, eq.(30)]. The integral in (9) can be computed by expanding the polynomial $F_{\lambda_{u}}\left(\frac{x(1+y)}{\alpha_{r u}}\right)^{U}$.

\subsection{High-SNR performance analysis}

To give further insights on the diversity performance, we provide the asymptotic analysis for the outage probability in the high-SNR scenario. In particular, we assume that, for each $u$, with constant ratio $\alpha_{s r} / \alpha_{r u} \rightarrow \kappa_{u}$, where $\kappa_{u}$ is a constant, as $\alpha_{s r}$ and $\alpha_{r u}$ tend to infinity, and the transmission power of the interferers is fixed.

It has been pointed out that the McLaurin series of the CDF of $\gamma_{U B}=\min \left(\gamma_{r}, \gamma_{\star}\right)$ has the same non-zero coefficient as that of the CDF of $\gamma$ [6]. Therefore, the asymptotic expression of the end-toend outage probability can be written as:

$$
F_{\gamma}^{\infty}(x)=F_{r}^{\infty}(x)+F_{\star}^{\infty}(x)-F_{r}^{\infty}(x) F_{\star}^{\infty}(x)
$$

where $F_{r}^{\infty}$ and $F_{\star}^{\infty}$ denote the asymptotic CDF expressions of the SINR for the source-relay channel and the relay-user channel, respectively.

Considering that $\mathbf{H}_{i j} \mathbf{H}_{i j}{ }^{\mathrm{H}}$ is a Wishart matrix, $F_{r}^{\infty}$ can be evaluated by exploiting the asymptotic expression of the CDF of the largest eigenvalue of such matrices [7]:

$$
\begin{aligned}
F_{r}^{\infty}(x)= & \sum_{i=1}^{\varrho(\mathbf{A})} \sum_{j=1}^{\tau_{i} \mathbf{A}} \sum_{g=0}^{v t}\left(\begin{array}{c}
v t \\
g
\end{array}\right) \\
& \frac{\chi_{i j}(\mathbf{A}) \alpha_{\langle i r\rangle}^{g}}{\Gamma(j)} \frac{\Gamma_{t}(t) \Gamma(g+j)}{\Gamma_{t}(v+t)}\left(\frac{x}{\alpha_{s r}}\right)^{v t} .
\end{aligned}
$$

In (11) $\Gamma_{a}(b)=\prod_{k=1}^{a} \Gamma(b-k+1)$.

Similarly, $F_{\star}^{\infty}$ can be evaluated according to (8):

$F_{\star}^{\infty}(x)=\sum_{u=1}^{U} \sum_{i=1}^{\varrho\left(\mathbf{B}_{u}\right)} \sum_{j=1}^{\tau_{i} \mathbf{B}_{u}} \sum_{g=0}^{U p q} \frac{\left(\begin{array}{c}U p q \\ g\end{array}\right)}{U \kappa_{u}^{-U p q}}$

$\left(\frac{\Gamma_{q}(q)}{\Gamma_{q}(p+q)}\right)^{U} \frac{\chi_{i j}\left(\mathbf{B}_{u}\right) \alpha_{\langle i u\rangle}^{g}}{\Gamma(j)} \Gamma(g+j)\left(\frac{x}{\alpha_{s r}}\right)^{U p q}$.

In the high-SNR regime $\alpha_{s r} \rightarrow \infty$. Thus the dominant term in (10) is that with the smallest exponent, namely, $v t$ or $U p q$. It follows that the asymptotic outage probability in the high-SNR regime can be written as:

$$
F_{\gamma}^{\infty}(x) \approx \begin{cases}F_{r}^{\infty}(x), & v t<U p q \\ F_{r}^{\infty}(x)+F_{\star}^{\infty}(x), & v t=U p q \\ F_{\star}^{\infty}(x), & v t>U p q .\end{cases}
$$


Such expression indicates that the diversity order of the system depends on $\min (v t, U p q)=$ $n_{r} \min \left(n_{s}, U n_{d}\right)$. Also, the diversity order is not degraded by the CCI received either at the relay or at the users.

\section{NUMERICAL RESULTS}

In this section the outage probability is presented as the number of antennas, the interference power level, and the number of the users vary. The analytical result in (6) is verified through Monte Carlo simulations. The path-loss exponent is set to $a_{i j}=$ 2.5 for all transmit-receive pairs. The number of interferers at any node is equal to 6 . The distances between the relay and each user is set to $d_{r u}=$ $(0.4+0.1 u) d_{s r},(u \in\{1, \ldots, U\})$. The distances between the interferers $\mathcal{S}_{r}$ and the relay, and between the interferers $\mathcal{S}_{u}$ and the user, are assumed to be, respectively, $d_{k r}=[0.9+0.1 k] d_{1 r}, k \in\{2,3,4,5,6\}$ and $d_{l u}=(0.9+0.1 l) d_{1 u}, l \in\{2,3,4,5,6\}$.

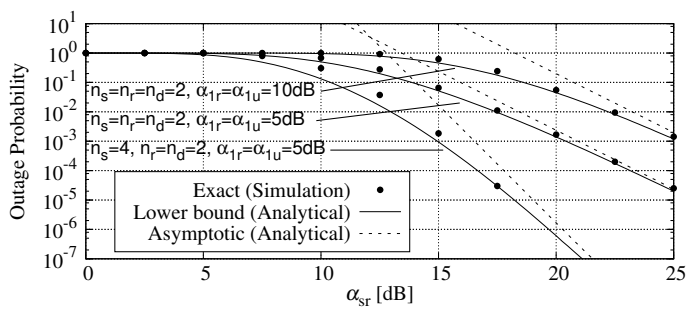

Figure 1: Outage probability of the systems with various antenna numbers and interference power, when $U=3$.

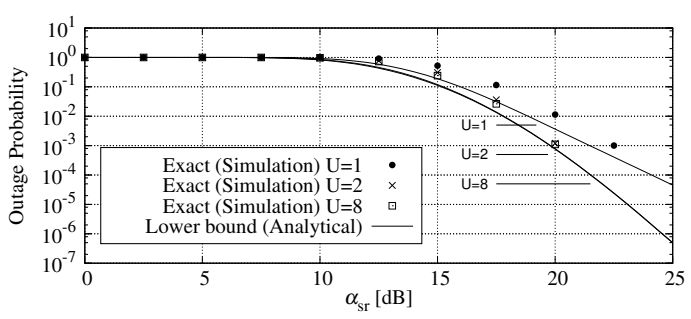

Figure 2: Outage probability of the systems with various number of users, when $n_{s}=4, n_{r}=n_{d}=2$ and $\alpha_{1 u}=10 \mathrm{~dB}$.

Fig. 1 compares the derived lower bound and the asymptotic expressions of the outage probability with the exact numerical results for $U=3$. The outage threshold is set to $x=5 \mathrm{~dB}$. We observe that our lower bound is very tight with respect to the Monte Carlo simulations, especially for high SNR. The presented asymptotic curves also match well with the numerical results in the high-SNR regime. Moreover, the diversity gain of the system, which in this case is equal to $n_{r} n_{s}$, determines the slope of the asymptotic curves.
Fig. 2 shows that our analysis holds also for quite large values of $U$ : curves have been obtained for $n_{s}=4, n_{d}=n_{r}=2$, thus for $U>2$ the system performance is unchanged.

\section{CONCLUSIONS}

We investigated the outage probability of dual-hop MIMO relay networks where AF is used for traffic relaying and hop-by-hop beamforming is applied. We derived a closed-form lower bound and an asymptotic expression of the outage probability when users are scheduled according to the PFS scheme. Our results are validated through numerical simulations. The results provide valuable insights on the design of practical multiuser dual-hop networks. Future work will address different multiuser scheduling strategies.

\section{ACKNOWLEDGEMENTS}

This work was supported by the European Union through the Xhaul project (Horizon2020-ICT671598).

\section{References}

[1] J. G. Choi and S. Bahk, "Cell-throughput analysis of the proportional fair scheduler in the singlecell environment," in IEEE Trans. Veh. Technol., Vol. 56, No. 2, Mar. 2007.

[2] P. A. Dighe, R. K. Mallik and S. S. Jamuar, "Analysis of transmit-receive diversity in Rayleigh fading," in IEEE Trans. Commun., Vol. 51, No. 4, Apr. 2003.

[3] G. Amarasuriya, C. Tellambura, and M. Ardakani, "Performance analysis of hop-by-hop beamforming for dual-hop MIMO AF relay networks," in IEEE Trans. Commun., Vol.60, No. 7, July 2012.

[4] U. Bletsas, H. Shin, and M. Z. Win, "Cooperative communications with outage-optimal opportunistic relaying," in IEEE Trans. Wireless Commun., Vol. 6, No. 9, Sep. 2007.

[5] S. Zhou, G. Alfano, C.-F. Chiasserini, and A. Nordio, "Outage probability of beamforming for multiuser MIMO relay networks with interference," Technical Report, https://www.dropbox.com/s/krwblpsdafzoqc0/TechRep.pdf?dl=0.

[6] H. Phan, T. Q. Duong, M. Elkashlan, and H. J. Zepernick, "Beamforming amplify-and-forward relay networks with feedback delay and interference," in IEEE Signal Process. Lett., Vol. 19, No. 1, Jan. 2012.

[7] S. Jin, M. R. McKay, X. Gao, and I. B. Collings, "Asymptotic SER and outage probability of MIMO MRC in correlated fading," in IEEE Signal Process. Lett., Vol. 14, No. 1, Jan. 2007. 